\title{
The Current Situation and Consideration of the Training of Nursing Talents in Traditional Chinese Medicine
}

\author{
Bei Wang ${ }^{1}$, Haiyun Gai ${ }^{2, *}$ \\ ${ }^{1}$ Shaanxi University of Chinese Medicine, China. \\ ${ }^{2}$ Department of Nursing, Xi' an Traditional Chinese Medicine Hospital, Shaanxi, China.
}

How to cite this paper: Bei Wang, Haiyun Gai. (2021) The Current Situation and Consideration of the Training of Nursing Talents in Traditional Chinese Medicine. International Journal of Clinical and Experimental Medicine Research, 5(4), 425-430.

DOI: 10.26855/ijcemr.2021.10.001

Received: July 2, 2021

Accepted: July 26, 2021

Published: August 6, 2021

*Corresponding author: Haiyun Gai, Department of Nursing, Xi'an Traditional Chinese Medicine Hospital, Shaanxi, China.

Email: 2548513941@ qq. com

\begin{abstract}
In clinical practice, nurses directly provide patients with traditional Chinese medicine (TCM) nursing technical services. At the same time, TCM nursing plays an important role in disease prevention, chronic disease management and community nursing, which is closely related to the health rights and life safety of the people. In order to accurately meet the growing diversified health needs of the people in the new era, strengthen the discipline construction of traditional Chinese medicine nursing, give full play to the characteristics and advantages of traditional Chinese medicine nursing, and promote the application of appropriate technologies of traditional Chinese medicine in nursing work, we should pay attention to the training of nursing staff and improve the traditional Chinese medicine nursing ability of traditional Chinese medicine nurses, so as to provide high-quality and Standardized TCM nursing service. This paper summarizes the current situation of the training of nursing talents in TCM, and considers the problems existing in the training of nursing talents in TCM.
\end{abstract}

\section{Keywords}

Traditional Chinese Medicine Nursing, Training Status, Personnel Training, Overview

\section{Introduction}

Nursing of traditional Chinese medicine (TCM) is an important part of the cause of TCM and an indispensable force for the inheritance and development of the cause of TCM. In recent years, the national health department issued a series of policies such as "National Nursing Development Plan (2016-2020)", "opinions on strengthening TCM Nursing work", "guidelines for issuing TCM Nursing work in TCM Hospitals" and a series of policies to encourage and require the training of excellent TCM nursing talents, give full play to the advantages of TCM nursing characteristics, improve the nursing effect of TCM, and standardize the nursing behavior of TCM [1-3].

In the outline of the National Nursing Development Plan (2016-2020), the Health and Family Planning Commission mentioned that TCM medical institutions and TCM departments of general hospitals and specialist hospitals should actively carry out syndrome differentiation nursing and specialty nursing with TCM characteristics, and innovate TCM nursing model. improve the nursing level of TCM [1]. TCM nursing not only has the theoretical advantages of holistic view, syndrome differentiation nursing, giving priority to prevention and paying attention to health preservation and rehabilitation, but also has the TCM nursing technology of "simplicity, convenience, 
cheapness and experience”, which makes it quickly recognized by patients in clinical development [4]. However, with the development of TCM nursing, the demand of the people for it is also increasing, and the existing TCM nursing resources cannot meet the needs of the people. the need to give full play to the role of TCM nursing in disease treatment, prevention and treatment of chronic diseases, rehabilitation promotion, health care and so on [5]. Sufficient TCM nursing talents can promote the development of TCM nursing and better meet the health needs of the people. The current situation of TCM nursing personnel training is summarized as follows.

\section{The existing problems of TCM nursing service}

\subsection{The shortage of nursing human resources}

Compared with western medicine nursing technology, TCM nursing technology operation is complex, time-consuming, and needs more nursing staff. Wei Lin and others pointed out that reasonable allocation of nursing human resources can reduce the incidence of adverse events, improve the quality of nursing, and increase the satisfaction of patients and nurses [6]. Chen Lili conducted a survey on nursing human resources in 805 TCM medical institutions in February 2019 with a self-designed questionnaire. The average bed-to-nurse ratio of 805 hospitals was $1 \mathrm{RV} 0.38$, including 271 tertiary hospitals, with an average bed-to-nurse ratio of 10.39, and 534 secondary hospitals, with an average bed-to-nurse ratio of $1 \mathrm{RV} 0.37$ [7]. It is lower than the requirement put forward by the health department in the "guidance on promoting the Reform and Development of Nursing Service Industry" [8] that the ratio of health care to health care should not be less than 1.25 by 2020 . At the same time, there are some problems, such as unreasonable professional title structure, unstable nursing team and so on. This is basically consistent with the conclusions drawn by scholars when they investigated the human resources of TCM nursing in Shanxi Province [9], Gansu Province [10], Tianjin [11] and Chongqing [12].

\subsection{The connotation of service is not deep enough.}

At present, only $26.72 \%$ of nurses in TCM institutions have TCM education background [7], which is higher than $24.71 \%$ in 2014 [13]. As a result, the TCM nursing skills and syndrome differentiation nursing level of some TCM nurses need to be improved. The research of Xu Wei and others shows that the development rate of TCM nursing service in TCM hospital is relatively high, but only the development rate of TCM nursing operation technology reaches $63 \%$, and the development rate of other items is not more than $40 \%$. For example: TCM emotional nursing, TCM health care and other nursing services, and the service target is limited to internal medicine patients and patients with mild illness, do not give full play to the potential of TCM nursing [14]. The investigation results of Wang Di and others also show that there are differences between the current TCM nursing service projects and the needs of inpatients in diet guidance, emotional nursing, TCM nursing, rehabilitation nursing, health care and so on. The development of TCM nursing service still exists the phenomenon of "across the board", which cannot meet the needs of specialties, and the quality of TCM nursing needs to be further improved [15].

\section{Current situation of nursing staff training in TCM}

\subsection{The training content, teaching materials and teacher training}

Teachers and contents are decided by each hospital. Due to the different development level of medical education in different places, the composition of the teaching staff varies greatly, but they are mainly taught by clinical nurses [7], and the teaching contents include TCM theory, TCM nursing technology and nursing routine of common diseases, etc. The main way of assessment is the combination of theoretical examination and practical skill examination.

In order to improve the service ability and level of newly recruited nurses in TCM hospitals, to guide all localities to standardize the training of newly recruited nurses in TCM hospitals, the State Administration of TCM issued the training syllabus (trial) for newly recruited nurses in traditional Chinese medicine hospitals in May 2018 (hereinafter referred to as the outline), which defines the training objectives, training time, training contents and assessment methods ${ }^{[16]}$. In order to put the training work into practice, the Nursing Branch of the Chinese Society of TCM, in accordance with the spirit of the State Administration of TCM, organized Zhang Suqiu and others to compile the training course for newly recruited Nurses in TCM Hospitals, which was published in April 2019 [17]. Therefore, when Chen Lili conducted a survey in February 2019, there was still a lack of unified teaching materials for the training of new nurses in various TCM medical institutions, and the starting point, depth, breadth and course subjects of training in different hospitals were different, and the training effect was lack of reasonable and standar- 
dized evaluation [7].

\subsection{The syllabus of teaching}

The methods requires combination of theoretical knowledge training and clinical practice ability training to train nurses, which can be trained by classroom teaching, group discussion, clinical rounds, operation demonstration, situational simulation, case nursing and other training methods [16]. Zhang Peijia's research results also show that the related research on teaching methods has become the focus of the training of new nurses [18]. In recent years, many researchers have adopted a variety of teaching methods in training nurses, such as reverse classroom, micro-class, mu class, situational exercise and so on. Wang Qing uses online and offline mixed learning mode to train new nurses. The experimental group is not only better than the control group in theory and skill operation examination, but also has better autonomous learning ability [19].

\subsection{Training ways and time}

Arrangements for TCM nurses, the main way to train TCM nurses is in-hospital training. There are few training bases for TCM nurses, and most of them focus on the training of backbone nursing talents and specialist nurses. The centralized training for new nurses is mainly carried out in a short time, and most of the training time for enrolled nurses is the morning shift or rest time, and the training is lack of standardization and systematization. This kind of training makes nurses, especially those who graduated from non-Chinese medicine colleges, unable to master the relevant knowledge of TCM and apply it to clinical practice [20].

\section{The existing problems of training TCM nurses}

\subsection{The training content cannot meet the needs of nurses}

Many investigations have found that TCM nurses have a high demand for training, while nurses at different levels have different needs for training content [21-23]. However, at present, when training nurses, hospitals pay less attention to the level and needs of the training objects. The key word "nurse training" is searched in the knowledge net and Wanfang database. From the search results, there are more literature studies on the training of new nurses, but less attention is paid to the training of new nurses. At present, the continuing education for nurses is based on professional skills credit training, and the training courses for nurses at all levels are basically the same, lack of targeted training courses, and do not take into account the growth needs of nurses.

\subsection{Clinical teaching is mainly based on experiential teaching}

When clinical training seniority nurses, one-to-one teaching is generally adopted, and the teaching teachers are mainly senior nurses. The teaching content is mainly clinical problems, which is random and accidental, and the ways to solve the problems are individuals with teachers, and lack of evidence-based evidence, which is not conducive to the cultivation of scientific research thinking of junior nurses.

\subsection{There is a lack of comprehensive effect evaluation indicators for the evaluation of training effec- tiveness, which is mainly based on the combination of theoretical examination and practical skills as- sessment}

The outline requires the process evaluation and completion assessment of the training objects, which mainly includes the evaluation of medical ethics, professional accomplishment, humanistic care, communication skills, theoretical learning and clinical practice. The stage-by-stage examination of basic theory training and the assessment of professional theories and skills of various specialties, and the graduation examination includes the assessment of theoretical knowledge and clinical practice ability [16]. Some scholars believe that [24] the training results can be evaluated by scientific research achievements and awards, but the purpose of training TCM nurses is to provide patients with high quality and safe TCM nursing services and to meet the health needs of patients, promote the development of TCM nursing. TCM nursing for patients cannot only prevent diseases and promote rehabilitation, but also play a positive role in health care, but these effects cannot be evaluated by a single index.

\section{Thinking}

\subsection{The establishment of a complete TCM}

Nursing theoretical system is the cornerstone of the training of TCM nurses. As the nursing theory of TCM is 
contained in the books of TCM, it is necessary for nursing workers to dig into the classics of TCM and dig out the nursing theory of TCM to form a complete theoretical system to define the professional orientation. Ma Xueling and others think that we should excavate and sort out the connotation of TCM nursing in the classics, improve nursing students' TCM cultural literacy and the ability to guide clinical practice by using classical theories and methods of TCM, so as to lay the foundation for the clinical nursing work of TCM [26]. In addition, Jiao Wenbo and others sorted out and summarized the nursing methods in Huangdi Neijing, summed up the nursing methods of life, diet and emotion, in order to expand the coverage of clinical TCM nursing, to further meet the needs of patients for TCM nursing to provide theoretical guidance [27].

\subsection{Adopt multi-channel, hierarchical and long-term training}

At the present stage, a number of training bases with advantages and characteristics of TCM nurses have been put into use. In the future, we can also explore the mode of joint training in colleges and universities, let the training subjects receive systematic theoretical education of TCM, and cultivate the dialectical thinking of TCM, so as to guide to provide dialectical nursing for patients in clinic.

At the same time, the training of nurses should not only stay in induction training, but also formulate long-term training contents according to the training needs and growth requirements of nurses at different levels. The study shows that the application of hierarchical training model can improve primary nurses' basic thinking, basic theories and skills of TCM nursing, and enable intermediate nurses to master the abilities of syndrome differentiation and health preservation and rehabilitation of TCM. so that expert nurses TCM nursing standardization research, TCM nursing evidence-based practice, teaching and other aspects to achieve results, through hierarchical training, TCM nursing operation technology can be widely carried out, promoted the development of TCM nursing scientific and technological achievements [23-25]. According to the requirements of the outline, the training objectives of new nurses can be established according to the characteristics of hospitals and departments, and the needs of training objects should be taken into account in order to improve the professional identity and core competence of new nurses [28]. Different training objectives should be set for the training of different types of TCM nurses, such as management type, specialty type, teaching type, research type and so on, and an echelon of TCM nursing talents with reasonable structure, strong professionalism and high quality should be trained at different levels.

\subsection{Combination of multiple teaching methods}

In order to ensure the teaching quality, we can make full use of online teaching, group discussion, situational exercises and so on. There are many research achievements on teaching methods, so we can choose appropriate teaching methods according to the characteristics of teachers and training objects. For example, we can adopt a practical teaching model that integrates medical record analysis, role-playing and real-person drilling. This model is helpful to improve the trainees' communication ability, dialectical nursing ability, practical ability and learning enthusiasm [29]. Plum blossom and others teach trainee nursing students through online TCM nursing service scripts, which is helpful to improve nursing students' thinking ability of syndrome differentiation and nursing cognition of TCM [30]. Chen Hongtao believes that peer education helps to improve the effect of trainee nursing students' TCM characteristic nursing technology training [31].

\subsection{Select the effect evaluation index with wide coverage}

The effect of TCM nursing service cannot be assessed by a simple scale, and simple methods such as examinations cannot be used in the assessment of TCM nurses. Some scholars will take the Kirschner model of multi-level effect evaluation as the guidance, using the methods of questionnaire survey, written test, scenario simulation and semi-structured interview to evaluate the effect of trained nurses from four levels: response layer, learning layer, behavior layer and result layer [32]. However, there is no unified evaluation standard for the evaluation index of training effect, which needs to be explored in the future work.

\section{Summary}

TCM nursing has attracted the attention of the people because of its unique advantages in disease prevention, promotion of rehabilitation, health care and other aspects, and the health department is also vigorously promoting the development of TCM nursing. However, there is still a large gap between TCM nursing and western medicine nursing. As an important cornerstone to promote the development of the discipline, the training of TCM nursing talents should be paid attention to. 


\section{References}

[1] National Health and Family Planning Commission. Notice on issuing the National Nursing Development Plan (2016-2020) [EB/OL]. 2016-11-24. Http://www.nhc.gov.cn.

[2] State Administration of traditional Chinese medicine. Opinions on strengthening nursing work of TCM [EB/OL]. 2013-07-26.www.satcm.gov.cn.

[3] State Administration of traditional Chinese medicine. Notice on issuing guidelines for traditional Chinese medicine Nursing in traditional Chinese medicine Hospitals (for trial implementation) [EB/OL]. 2010-07-28.www.satcm.gov.cn.

[4] Sun Kaili, Hu Hui, Wang Ling, et al. (2017). Study on the advantages and limitations of the development of clinical nursing of TCM [J]. Shi Zhenguo Medical Medicine, 2017 Journal ,28(06): 1411-1413.

[5] Hu Yanmin, Li Haiyu, Xue Ming, et al. (2017). The present situation and countermeasures of human resources development in public traditional Chinese medicine hospitals in China [J]. Chinese Journal of Traditional Chinese Medicine Information, 2017 Journal, 24(10): 5-9.

[6] Wei Lin, Liu Yangchen, Zhu Jing, et al. (2017). Allocation and evaluation of nursing human resources in traditional Chinese medicine hospitals based on nursing workload [J]. Nursing Management in China, 2017, 17(01): 95-99.

[7] Chen Lili, Zhang Suqiu, Wang Danqing, Zhou Jiaomei. (2019). Investigation on the current situation of nursing human resources in 805 traditional Chinese medicine medical institutions [J]. Nursing Management in China, 2019, 19(10): 1456-1460.

[8] Guidance on promoting the reform and development of nursing service industry [J]. Nursing Management in China, 2018, 18(07): 865-867.

[9] Li Mei, Chai Yan, Song Huawen. (2015). Current situation and analysis of nursing human resource allocation in 33 secondary traditional Chinese medicine hospitals in Shanxi Province [J]. Nursing Research, 2015, 29(22): 2774-2775.

[10] Yang Jingyu, Wang Rongjuan, GE Yonghong, et al. (2017). Analysis on the stock of human resources of traditional Chinese medicine under the characteristic medical reform in Gansu Province [J]. Chinese Journal of Traditional Chinese Medicine Information, 2017 pr, 24(03): 9-13.

[11] Yang Xuan, Wang Yuling, Bai Xiuli. (2016). Investigation and analysis on the current situation of nursing team construction in 19 traditional Chinese medicine hospitals in Tianjin [J]. Nursing Management in China, 2016 pr, 16(02): 156-159.

[12] Yang Xijuan, Zhao Xiwei, Zhao Yang, et al. (2016). Development status and countermeasures of nursing human resources in traditional Chinese medicine hospitals in Chongqing [J]. Nursing Management in China, 2016 Regent, 16(09): $1244-1248$.

[13] Zhang Suqiu, Zhou Jiaomei, Chen Lili. (2015). Investigation, analysis and thinking on the development of nursing discipline in traditional Chinese medicine [J]. Nursing Management in China, 2015, 15(06): 642-645.

[14] Xu Wei, Wang Jialan, Wang Yili, et al. (2018). Investigation and Analysis on the current situation of traditional Chinese medicine Nursing Service in Grade 3A Hospital of traditional Chinese medicine [J]. Hospital Management in China, 2018, 38(04): 75-77.

[15] Wang Di, Fang Xiuping, Zuo Yi, et al. (2019). Analysis on the difference between supply and demand of traditional Chinese medicine nursing service for inpatients in public Grade 3A traditional Chinese medicine Hospital [J]. Nursing Research, 2019, 33 (19): 3409-3412.

[16] Office of the State Administration of TCM. Circular of the Office of the State Administration of traditional Chinese medicine on issuing the training syllabus for newly recruited Nurses in traditional Chinese medicine Hospitals (trial) [EB/OL]. 2018-05-14.www.satcm.gov.cn.

[17] Zhang Suqiu, Liu Xiangdi, Guo Jing. (2019). Training course for newly recruited Nurses in traditional Chinese medicine Hospitals [M]. Beijing: published by China traditional Chinese medicine Publishing House, 2019

[18] Zhang Peijia, Peng Wentao, Liao Xin. (2019). Co-word analysis of literatures related to the training of new nurses in China [J]. Nursing Education in China, 2019, 16(10): 754-758.

[19] Wang Qing, Lin Yanjuan, Hu Rong, et al. (2019). Application of blended learning in the training of new nurses in ICU [J]. Chinese Nursing Education, 2019, 16(10): 742-745.

[20] Zhou Xiaoyun, Cui Yi, Zhang Yali. (2015). Qualitative study on nurses' experience of nursing techniques in traditional Chinese medicine [J]. Journal of Nursing, 2015, 30(09): 37-Qing

[21] Li Hong, Zhang Lingling, Chen Guilan, et al. (2015). Current situation and countermeasures of nursing continuing education in traditional Chinese medicine [J]. Journal of Nursing, 2015 pr, 30(07): 57-59.

[22] Xu Cui, Wu Peixiang, Xiao Liyun, et al. (2019). Research progress on the current situation of traditional Chinese medicine nursing training for nurses [J]. China Medical Herald, 2019pr, 16(16): 50-53.

[23] Guo Xiujun, Zhang Huiling, Cao Cong, et al. (2017). Study on the Construction of Nursing talents training course in traditional Chinese medicine Hospital [J]. Nursing Education in China, 2017, 14(06): 422-425.

[24] Zhou Jianmei, Wang Xiaodong, Zhang Yali. (2013). Practice and thinking of personnel training in nursing discipline of traditional Chinese medicine [J]. Journal of Nursing, 2013, 28(21): 48-50. 
[25] Yuan Wei, Yang Guihua, Zhou Aixia, et al. (2014). Echelon construction and practice of traditional Chinese medicine nursing talents in general hospital [J]. Journal of Nursing, 2014 Jing, 29(13): 35-Qing.

[26] Ma Xueling, Liu Jing, Luo Hui, et al. (2019). Take Treatise on febrile Diseases as an example to explore the train of thought of traditional Chinese medicine nursing classic teaching in colleges and universities of traditional Chinese medicine [J]. Nursing Education in China, 2019 Regent, 16(02): 130-132.

[27] Jiao Wenbo, Gai Fengchun, Qin Tingzheng, et al. (2019). Exploration of traditional Chinese medicine nursing methods in Huangdi Nei Jing [J]. Journal of Changchun University of Traditional Chinese Medicine, 2019, 35(04): 795-797.

[28] Qi Xiangxiu, Li Ke, Cheng Xianjuan, et al. (2019). The practical effect of two-way demand-oriented training for new nurses [J]. Nursing Education in China, 2019 Regent, 16(10): 729-732.

[29] Wang Yuncui, Zhou Huifang, Shu Jing, et al. (2014). Practical teaching reform and effect analysis of basic Nursing of traditional Chinese medicine [J]. When Zhenguo Medical Medicine, 2014, 25(01): 200-201.

[30] Plum Blossom, Lu Gendi. (2018). Effect of online traditional Chinese medicine nursing service script on trainee nursing students' thinking ability of syndrome differentiation and nursing cognition of TCM [J]. PLA Journal of Nursing, 2018, 35(17): 70-72, 76.

[31] Chen Hongtao, Gao Juan, Wang Qing, et al. (2017). Effect of peer education in clinical teaching of characteristic nursing technology of traditional Chinese medicine [J]. Journal of Nursing, 2017, 32 (19): 11-13.

[32] Huang Xueyan, Zhang Yin, Feng Ying, et al. (2018). Application of Kirschner model in evaluation of nursing training effect of traditional Chinese medicine in Hangzhou [J]. Chinese Journal of Nursing, 2018, 53(01): 71-75. 